\title{
¿SON LAS GRULLAS INDICADORAS DE LA RIQUEZA DE ESPECIES DE AVES ACUÁTICAS EN HUMEDALES EN EL ALTIPLANO MEXICANO?
}

\author{
EdgAR G. LÓPEZ-SAUT, ${ }^{1}$ RicARdo RODRÍGUEZ-ESTRELLA ${ }^{1}$ \& FELIPE \\ CHÁVEZ-RAMÍREZ ${ }^{2}$ \\ ${ }^{1}$ Centro de Investigaciones Biológicas del Noroeste S. C., Mar Bermejo No. 195, Col. Playa Palo de \\ Santa Rita Apdo. Postal 128; La Paz, BCS 23090, México, Tel:(52) (612) 123-8484 Fax:(52) (612) \\ 125-3625 \\ ${ }^{2}$ Conservation Programs Gulf Coast Bird Observatory, 103 Hwy 332 West Lake Jackson, TX 77566 \\ *<avez_g2002@yahoo.com.mx>
}

López-Saut, E. G., Rodríguez-Estrella, R. \& Chávez-Ramírez, F. 2014. ¿Son las grullas indicadoras de la riqueza de especies de aves acuáticas en humedales en el Altiplano Mexicano? Acta Zoológica Mexicana (n. s.), 30(2): 268-287.

RESUMEN. Los humedales son de los ecosistemas más productivos en el mundo y contienen una alta diversidad de especies. A pesar de esto, se encuentran amenazados por las actividades humanas que los modifican afectando su función hasta la desaparición de algunos de ellos, particularmente aquellos que se encuentran en zonas desérticas. Por lo anterior, es necesario generar herramientas que sean simples pero confiables para evaluar la calidad del humedal y su importancia para los grupos biológicos, con el fin de detectar tendencias y proponer medidas de conservación. Un ejemplo es utilizar bioindicadores que funcionen como indicadores de la riqueza de especies aves acuáticas. La Grulla gris (Grus canadensis) depende de los humedales en sus sitios invernales como refugio. Esta especie es considerada una especie bajo protección en México. El objetivo de este trabajo es analizar si puede ser un indicador de la riqueza de especies acuáticas para una evaluación rápida del humedal. Aunque no se encontró una correlación entre la presencia de las grullas y la riqueza de especies de aves acuáticas, sí se encontraron diferencias en la composición de especies y una mayor riqueza en los humedales donde están presentes las grullas. Los humedales no tuvieron similitud en la composición de especies, por lo que las grullas sirven de indicadoras para diferentes ensambles de aves acuáticas. Al hacer un análisis propuesto para identificar especies bioindicadoras, las grullas tienen un valor de 27.5 de un máximo de 32 . Por lo anterior las grullas pueden ser una especie indicadora de la riqueza de especies acuáticas. Deben hacerse estudios a largo plazo y evaluar la relación de las abundancias de las grullas con las abundancias de las aves acuáticas para reforzar los resultados aquí presentados.

Palabras clave: Bioindicador, grulla gris, México, humedales desérticos, desierto Chihuahuense, amenazados. 
López-Saut, E. G., Rodríguez-Estrella, R. \& Chávez-Ramírez, F. 2014. Are Sandhill cranes indicators of waterbirds species richness on wetlands of the Mexican Plateau? Acta Zoológica Mexicana (n. s.), 30(2): 268-287.

ABSTRACT. Wetlands are among the most productive ecosystems in the world and contain a high diversity of species. Despite this, wetlands are threatened by human activities because function is affected and some of them even disappear, particularly those in desert areas. Therefore, it is necessary to build simple tools but reliable for assessing the quality of a wetland and that can remark the importance of wetlands for biological groups in order to detect species population trends and to propose conservation strategies of wetlands and their biodiversity. An example is the use of particular species as bioindicators of species richness (e.g. of waterfowl). The Sandhill crane (Grus canadensis) depends on wetlands as wintering refuge sites. This crane is listed as a threatened species in Mexico. The aim of this paper is to analyze whether the Sandhill crane can be considered as an indicator of waterbirds' richness species in order to perform a rapid assessment of any wetland the crane uses. Although a correlation between of the presence of cranes and richness of waterbird species was not found a difference was observed in the composition of species and relatively greater species richness in wetlands where it cranes were present. Wetlands were not similar in species composition, thus cranes can be a useful indicator for different assemblages of waterbirds. Using the bio-indicator species analysis the Sandhill crane has a value of 27.5 from a maximum of 32. Therefore, the Sandhill crane can be considered as an indicator of waterbirds species richness in arid wetlands. Long-term studies should be done in order to evaluate the relationships between the abundances of cranes and the total abundance of waterbirds in order to evaluate the quality of wetlands in relation to abundance.

Key words: Bioindicator, sandhill crane, Mexico, arid wetlands, Chihuahua desert, species at risk.

\section{INTRODUCCIÓN}

Los humedales son de los ecosistemas más productivos en el mundo y contienen una alta diversidad de especies (Toledo 2006, Sánchez et al. 2007). Estos ofrecen servicios ecosistémicos tales como ser una fuente de agua, regular la temperatura en sus alrededores y tener una alta productividad que proveen de una gran variedad de alimento y materia prima (Toledo 2006, Sánchez et al. 2007). Los humedales se encuentran actualmente amenazados por las actividades humanas, principalmente los manglares y aquellos ubicados en zonas desérticas y semidesérticas (Frazier 1999, CONAGUA 2011). Es importante mencionar que más del $80 \%$ del territorio de México es desértico o semidesértico (Aguilar 2003, CONAGUA 2007), aunque estudios recientes hablan de un $65 \%$ (Verbist et al. 2010), lo que indica que hay una baja cantidad y disponibilidad de agua en más de la mitad del país. Los pocos humedales que hay en estas áreas son prioritarios para la vida silvestre y los asentamientos humanos, pero se encuentran amenazados por la sobreexplotación y cambio del uso de suelo. En estos ecosistemas los humedales son permanentes y temporales. Los temporales se forman después de la época de lluvias debido al efecto de las cuencas extensas en las partes bajas, que en el desierto Chihuahuense se les conoce como playas (Grünberger 2004).

Los asentamientos humanos y la agricultura se encuentran en expansión en las zonas desérticas y semidesérticas (SEMARNAT 2009), lo que produce una alta presión 
sobre los mantos acuíferos y los humedales en estas regiones, principalmente los temporales. La extracción de agua para las áreas urbanas y la agricultura afecta la permanencia de los humedales, su temporalidad, sus niveles de agua y su disponibilidad.

Los humedales, con un plan de manejo o sin él, son un refugio para numerosas especies de plantas y animales entre invertebrados, aves acuáticas y reptiles, algunos completamente dependientes de ellos para la reproducción o sólo durante la migración (Morgan \& Boy 1982, Hinojosa-Huerta et al. 2004, Cooper et al. 2006). Las presiones humanas sobre los humedales en zonas áridas y semiáridas tienen efectos negativos directos sobre la fauna que depende ellos, muy en especial a las aves acuáticas (Aguilar 2003, Cooper et al. 2006, Samraoui \& Samraoui 2008).

Las aves acuáticas comprenden tanto a los anátidos como a las especies de playeros, garzas y grullas, entre otras, que utilizan los humedales para alimentación o refugio durante el día o la noche. Incluyen especies migratorias que pasan el invierno o usan como sitios de descanso (sensu stopovers) los humedales en México durante su migración (Rappole et al. 2003). La pérdida o deterioro de estos sitios afecta la sobrevivencia invernal y la conectividad entre los sitios reproductivos y los migratorios, por lo que es importante identificar la condición de los humedales para su manejo y protección (Tankersley Jr. 2004).

Para determinar el estado de los humedales se pueden hacer diversas evaluaciones, desde aquellas que requieren muchos años de investigación y que estudian las variaciones poblacionales y el estado de salud de la fauna, hasta aquellas que buscan identificar rápidamente el estado del humedal (fast track), como son las generadas por RAMSAR y la UICN (Springate-Baginski et al. 2009). Estas evaluaciones de corto plazo son importantes para generar información suficiente con el fin de establecer las acciones necesarias para mantener y conservar los humedales y su biodiversidad de manera urgente, en cortos tiempos, pues de otra manera el deterioro será mayor y sus consecuencias irreversibles. Dentro de estas evaluaciones, el uso de especies indicadoras (o bioindicadores) es útil debido a que con su monitoreo se pueden detectar los cambios en el ecosistema y evaluar las acciones de manejo y conservación a corto plazo (Mikusiński et al. 2001, Fleishman et al. 2005, González et al. 2003, Drever et al. 2008).

Entre otros, Karr (1991) conceptualiza las características que debe tener una especie bioindicadora para evaluar la Integridad Ecológica para ríos en Estados Unidos. Los peces son un ejemplo por su estrecha relación con la estructura y calidad del humedal. Okes et al. (2008) establece que las aves son buenos indicadores de la calidad de un humedal debido a que son un grupo fácil de observar, se puede evaluar de forma sencilla su abundancia y composición, y son carismáticas, lo cual ayuda para la conservación y manejo de los humedales.

Dentro de las aves acuáticas, las grullas son una de las familias de aves que están más amenazadas (Meine \& Archibald 1996), pero a la vez su belleza, la facilidad de 
observación y por ser migratorias de interés común para más de un país, las hacen propicias para ser utilizada como especie bandera y paraguas para la protección de los humedales (Meine \& Archibald 1996, von Treuenfels 2006). En América se encuentran dos especies de grullas: la blanca (Grus americana) que debido a la pérdida de hábitat está en peligro de extinción, aunque su distribución actual no incluye a México (aunque se han presentado algunos reportes no confirmados de algunos avistamiento, no hay una prueba que aun visiten con regularidad los humedales de México) ya que sus poblaciones desaparecieron del país por cacería y pérdida de hábitat (Canadian Wildlife Service and U. S. Fish and Wildlife Service 2005, Howell \& Webb 1995), y la grulla gris (Grus canadensis), que después de acciones de conservación de los humedales y control de la cacería se encuentra actualmente aparentemente estable en sus poblaciones y en algunos casos en expansión (Kinzel et al. 1996, López-Saut et al. 2011).

La Grulla gris es una especie poco estudiada en México, no obstante que se ha identificado un conflicto entre los campesinos (Barceló et al. 2012) y las grullas debido a que se alimentan en los campos de cultivo durante el invierno. Y es paradójico que mientras se encuentra Bajo Protección Especial por la NOM-059-SEMARNAT2010 (SEMARNAT 2010) se considera una especie cinegética de acuerdo a la Ley General de Vida Silvestre (SEMANAP 1999, Contreras et al. 2001, SECTUR). La grulla gris es una especie migratoria que en México pasa el invierno principalmente en el desierto Chihuahuense (López-Saut et al. 2011) en Chihuahua, Durango, Coahuila, Nuevo León, Zacatecas y San Luis Potosí, por lo que depende de los humedales que se encuentran en estas zonas.

Para algunas Áreas de Importancia para la Conservación de las Aves (AICA), la presencia de las grullas fue clave para su designación por ser una especie que está en la NOM y estar estrechamente asociada a los humedales. Un ejemplo es la Laguna de Babícora que además fue establecida como un sitio RAMSAR y que alberga la mayor población de grullas gris en México (Arizmendi \& Márquez 2000, CONANP 2010).

Aunque la fuente principal de alimento para las grullas no se encuentra en los humedales durante el invierno, éstos son importantes pues se congregan en parvadas dentro de ellos durante el tiempo que dedican al descanso y durante la noche, por lo que los humedales les brindan protección, son zonas de refugio. Dada su talla y esta condición de agrupación son fáciles de observar en gran parte de los humedales del Desierto Chihuahuense. A pesar de su importancia ecológica y cultural (por ejemplo en el libro La Visión de los Vencidos se describe a la grulla como una de las señales que presagiaban la llegada de los españoles; León-Portilla 1999) es una especie poco estudiada en México. Por lo anterior, al igual que otras grullas, por ejemplo la grulla japonesa que es un símbolo importante para Japón, proponemos que puede ser reevaluada la grulla para ser como bioindicador y al conservar un humedal por su presencia pueda ser usada como una especie bandera para la conservación de los humedales del 
Desierto Chihuahuense, principalmente de aquellos de zonas desérticas que son de gran importancia para todas las aves migratorias durante el invierno. Para determinar su posible uso como especie indicadora del estado de los humedales nuestro objetivo fue determinar si existe alguna relación entre la presencia de la grulla gris y la riqueza de aves acuáticas durante su estancia invernal en el desierto Chihuahuense de México.

\section{MATERIAL Y MÉTODOS}

Durante el invierno de 2008-2009 se realizaron recorridos por tierra en 51 humedales en los estados de Chihuahua, Durango, Coahuila, Nuevo León, Zacatecas y San Luis Potosí, con el fin de registrar la presencia de las grullas (Fig. 1). Cada humedal se visitó temprano en la mañana o casi al atardecer para determinar la presencia de las grullas antes de que salieran del humedal o cuando regresaban a las zonas de descanso. En los humedales donde se encontraron rastros de la presencia de las grullas, pero no se registró visualmente, se realizó una sola visita y se registró el humedal como con presencia de grullas. Debido a que el desierto Chihuahuense tiene varios humedales temporales que son de gran importancia para las aves migratorias, se incluyeron para explorar si había una relación entre la riqueza de aves, la presencia de las grullas y los humedales temporales. Se consideraron humedales temporales aquellos que sólo permanecían durante la temporada o parte del invierno con agua. Para determinar la temporalidad, principalmente se usaron las observaciones de campo (presencia de campos de cultivos, zonas de uso rural, canchas de futbol, por mencionar algunas señales que indicaban que estaba seco anteriormente) y la información de la gente, porque la mayoría de los humedales en el área de estudio carece de información previa por ser temporales.

Previo a los conteos se realizaron visitas a varios humedales para contar a las aves y tener una idea del comportamiento de las mismas dentro del humedal. Para establecer el número de especies en los humedales se ubicó un punto de muestreo por humedal en un sitio desde donde se pudiera observar la mayor extensión del mismo. En los humedales más grandes se seleccionaron tres puntos de muestreo y se utilizó el punto con mayor número de especies para el análisis. El registro de las aves se hizo una vez por humedal, haciendo la observación durante 5 minutos durante los cuales se registraron todas las especies que estaban dentro del humedal, se utilizó este tiempo ya que los humedales era de tamaño pequeño y se podía observar todo el espejo por ser áreas planas sin vegetación, principalmente los humedales temporales. Los conteos se hicieron siempre por la mañana y por corto tiempo para evitar dobles conteos de los individuos en caso de moverse. Se pudo confirmar en varios humedales que se podían identificar a la mayoría de las especies de esta forma ya que era antes que dejaran el humedal principalmente especies que sólo lo usaban como descanso durante la noche. 


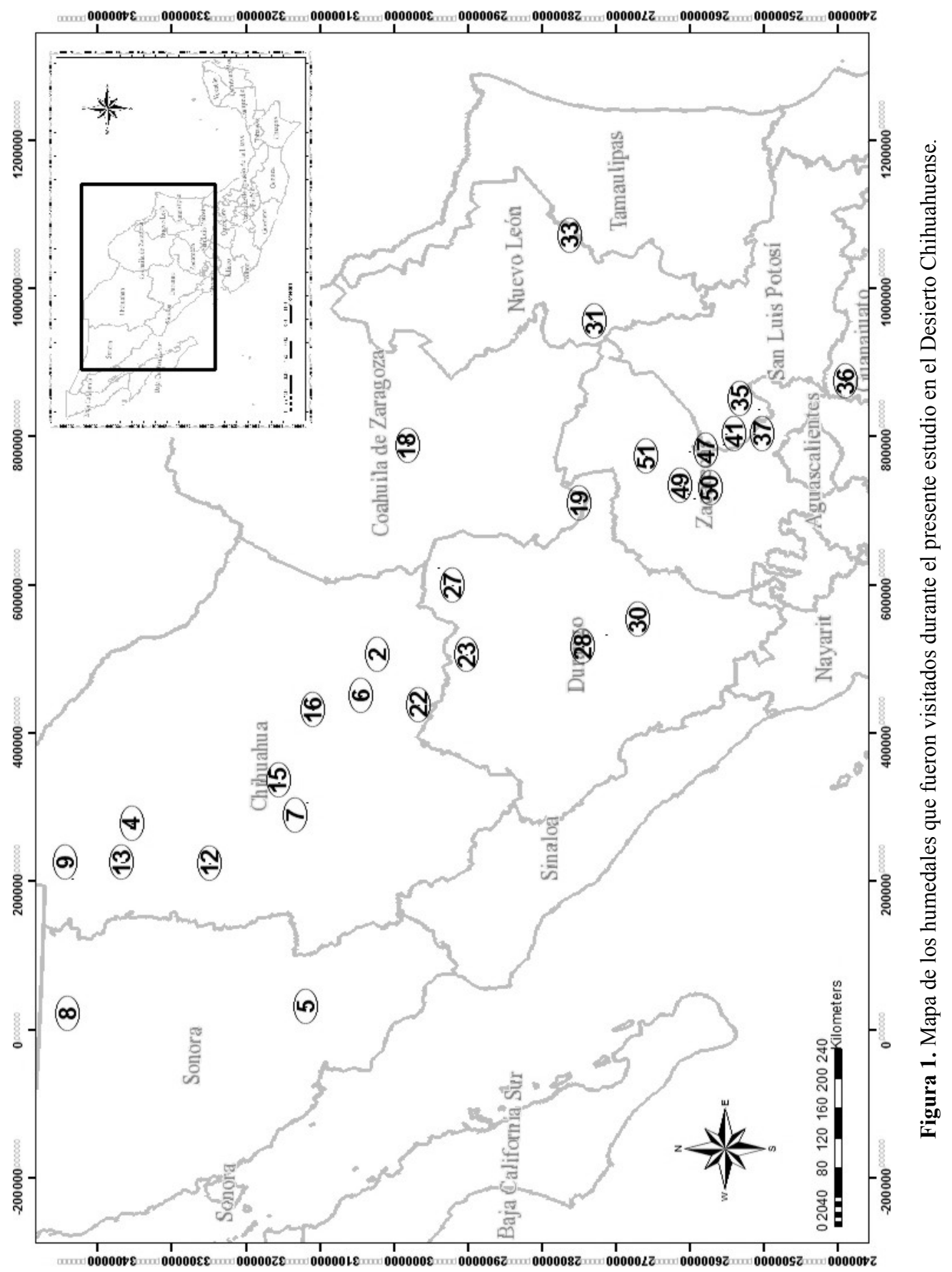


Se realizaron tres análisis de correlación, el primero entre la riqueza de especies de los humedales y la presencia de grullas, el segundo entre la riqueza de especies y la abundancia de grullas, y el tercero entre la riqueza de especies y la abundancia considerándola en categorías. También se hizo la correlación de la riqueza de especies por gremio (Anátidos, playeros y otras aves) y la presencia de grullas. Se generó una matriz de presencia y ausencia de cada una de las especies registradas en los humedales, con una columna de presencia y ausencia de grullas en los mismos humedales para realizar una prueba de G (Sokal \& Rohlf 1994) con el fin de determinar si cada especie estaría en un humedal asociada o no a la presencia de las grullas. Si el valor de $\mathrm{G}$ obtenido en la prueba es mayor al valor de $\chi^{2}$ de tabla a 0.05 significa que la distribución de las especies no es igual por lo que hay una relación con la presencia de las grullas.

Para determinar si los humedales se parecían entre sí en la composición de especies se utilizó el coeficiente de comunidad de Whittaker para establecer en una tabla los humedales más similares entre sí como complemento a la información generada por el índice de Jaccard, siguiendo la siguiente fórmula (Whittaker 1975):

$$
\mathrm{CC}=2 S_{a b} /\left(S_{a}+S_{b}\right)
$$

donde $S_{a b}$ es el número de especies en común entre los dos humedales, $S_{a}$ es el número de especies del humedal $a$ y $S_{b}$ es el número de especies del humedal $b$. Este índice compara los humedales para determinar el nivel de similitud entre los humedales con grullas y los que no tenían grullas.

También se hizo la prueba de similitud de Jaccard que nos permitió relacionar gráficamente los humedales más parecidos entre sí, utilizando la siguiente fórmula:

$$
\mathrm{Ij}=\mathrm{c} / \mathrm{a}+\mathrm{b}-\mathrm{c}
$$

donde a es el número de las especies en el humedal $a$, b es el número de especies en el humedal b y c el número de especies que comparten los humedales. El análisis se hizo usando el programa PAST.

Para evaluar si la grulla puede ser considerada como un bioindicador se utilizó la metodología propuesta por González et al. (2003). Para la evaluación se utilizaron los criterios de estado de conservación, endemismo, distribución geográfica, especialización a un hábitat, susceptibilidad de muestreo, facilidad de determinación y manipulación en campo, grado de conocimiento de su biología e historia natural y posibilidad de que refleje información del hábitat. Para generar la matriz de biología y la matriz de manejo, se utilizó para el estado de conservación la NOM-059-SEMARNAT-2010 y para el grado de endemismo y estacionalidad la información de Howell \& Webb (1995). Para la biología, distribución, hábitat y manejo se usó la 
información de Tacha et al. (1992), Meine \& Archibald (1996), Sharp et al. (2006) y López-Saut et al. (2011). Teniendo esta información se construyeron dos matrices, una biológica y otra de manejo, y para llenarlas se asignaron valores ponderados a cada criterio (ver el Apéndice 1 de González et al. 2003), para obtener un valor total final que se comparó con los propuestos por González et al. (2003).

\section{RESULTADOS}

Se registró la grulla gris en 30 de los 51 humedales (59\%; Fig. 1), de éstos 30, 11 (37\%) fueron humedales temporales. Sólo seis de los humedales donde no se registraron grullas fueron temporales. En resumen en total 17 humedales fueron temporales, es decir el $32 \%$ del total $(\mathrm{N}=51)$

En la figura 2 se observa que en los humedales donde hay presencia de grullas existe un relativo mayor número de especies que donde las grullas están ausentes. Se obtuvo un listado de 39 especies presentes en los humedales. Los anátidos fueron los que más especies presentaron con 13, seguidos de los playeros con 11. Las demás especies de aves acuáticas fueron las vadeadoras como las garzas, pelícanos, cormoranes, gaviotas y los buceadores. En el caso de los playeros (Charadriiformes) se registró un mayor número de especies en los humedales donde no se registraron grullas (Cuadro 1). En ninguna de las pruebas estadísticas realizadas se obtuvieron correlaciones significativas (Cuadro 2, 3, 4 y 5). En cuanto al índice de Whitaker se identificaron los humedales más similares entre sí (Cuadro 6), pero la mayoría de los humedales no tuvieron similitud entre ellos. La similitud entre los humedales es baja de acuerdo al índice de Jaccard (Fig. 3). Se encontró una relación significativas entre la riqueza de especies y la presencia de grullas $\left(\mathrm{G}=49.3045 \chi^{2}=43.77\right.$, g.l. $\left.=40, p<0.05\right)$.

En cuanto a la evaluación como bioindicador, se sumaron los valores de cada una de las matrices, siendo el total de la matriz de biología de 15.5 (valor máximo es 20) y para la matriz de manejo el valor obtenido fue de 12 (valor máximo es 12). Las suma de los dos valores da un total de 27.5 de un valor de 32 , que es el valor máximo para una especie bioindicadora propuesta por González et al. 2003 (Cuadros 6 y 7).

\section{DISCUSIÓN}

A pesar de que no existe una correlación clara entre las grullas y la riqueza de especies de los humedales, existe una diferencia en los grupos de aves presentes cuando hay grullas. La similitud entre los humedales no fue alta lo cual puede indicar una complementariedad entre los mismos por estar presentes diferentes especies en cada humedal, lo cual tendría como consecuencia tener mayor riqueza de especies en todos los humedales. Además, al ubicar los sitios que tienen mayor similitud geográficamente, estos se ubican en diferentes áreas del desierto Chihuahuense, es decir, que no se encuentran cercanos entre ellos. 


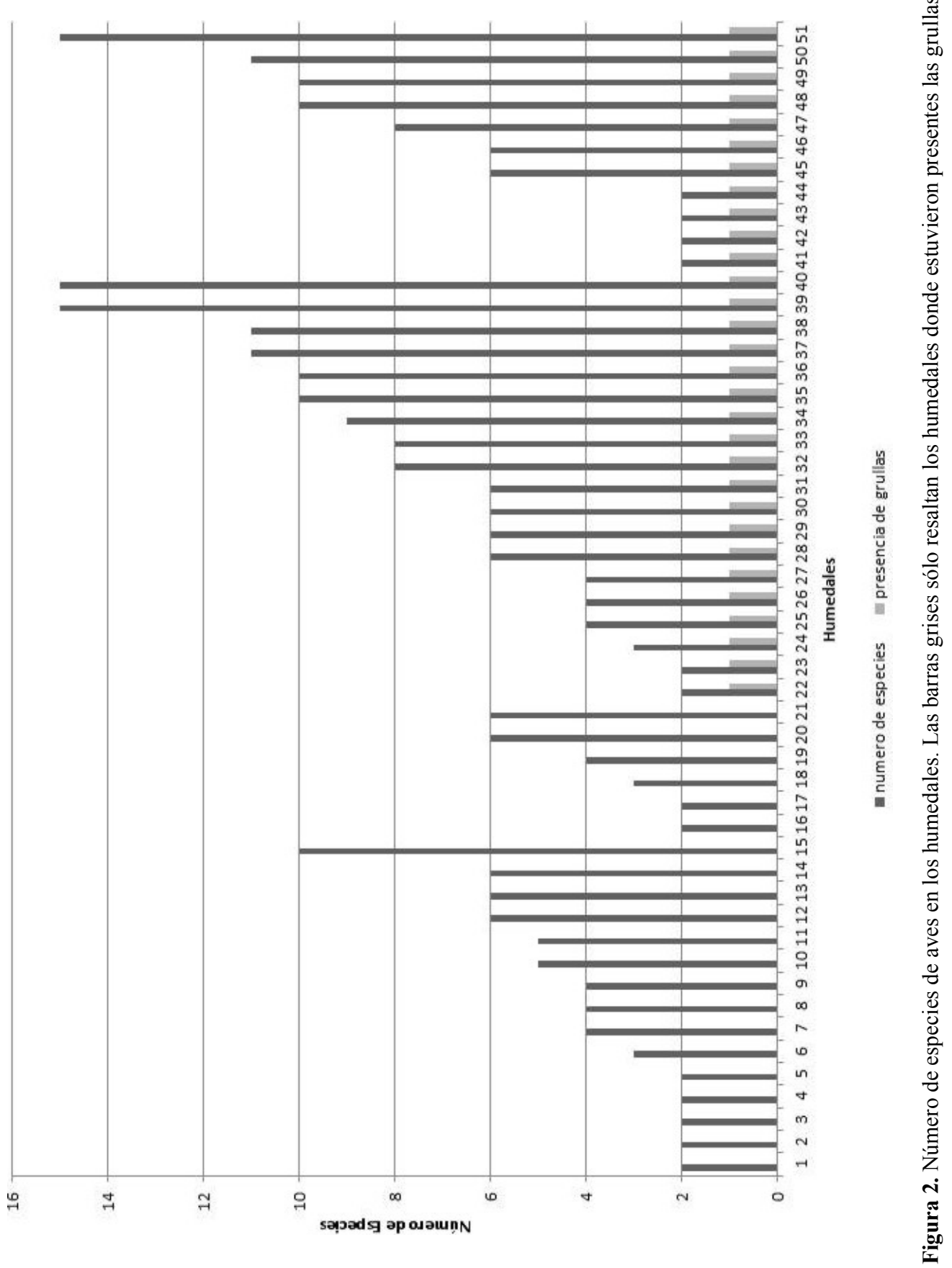


Acta Zool. Mex. (n.s.) 30(2) (2014)

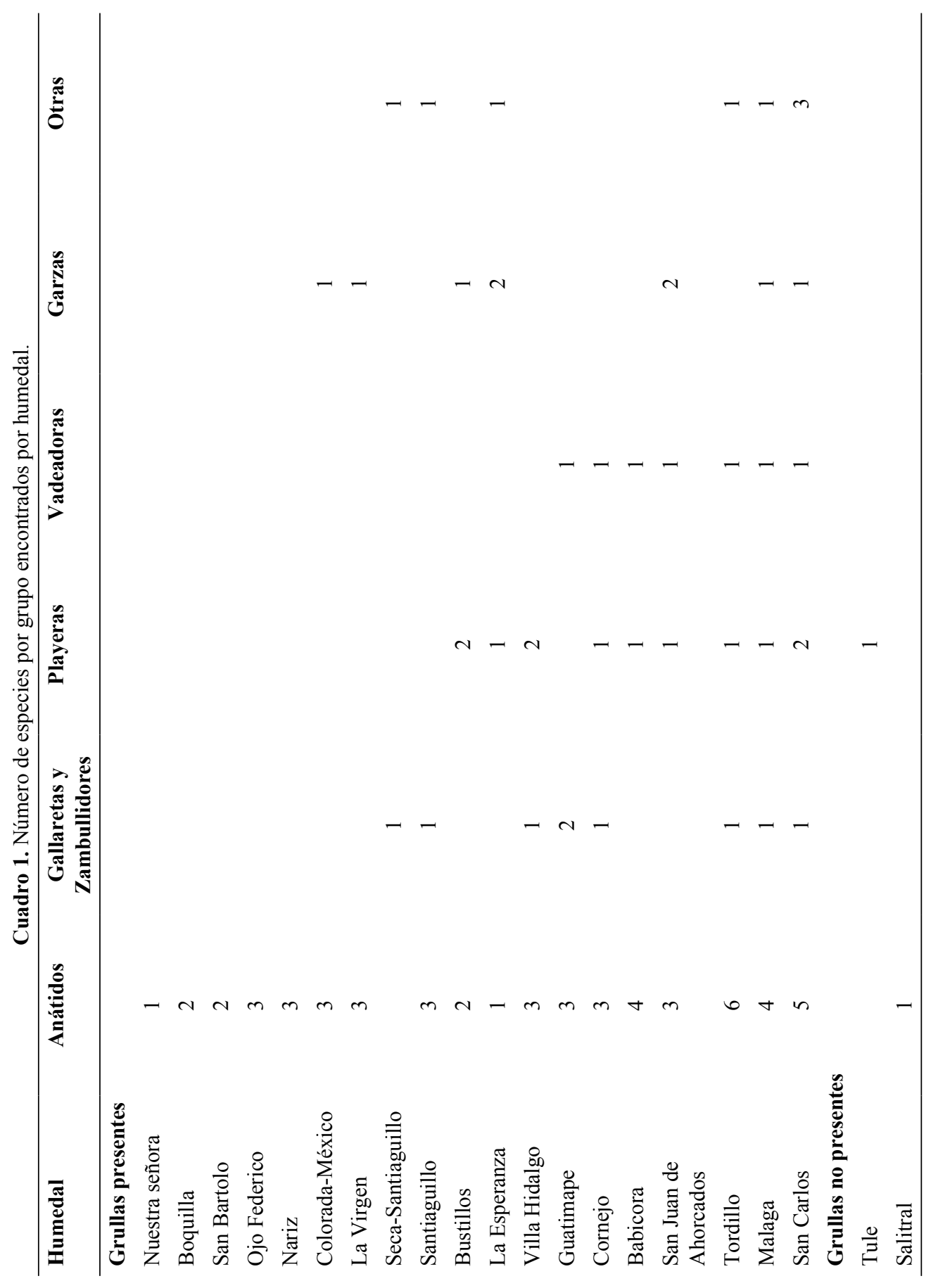


López-Saut et al.: La grulla como indicador de aves acuáticas

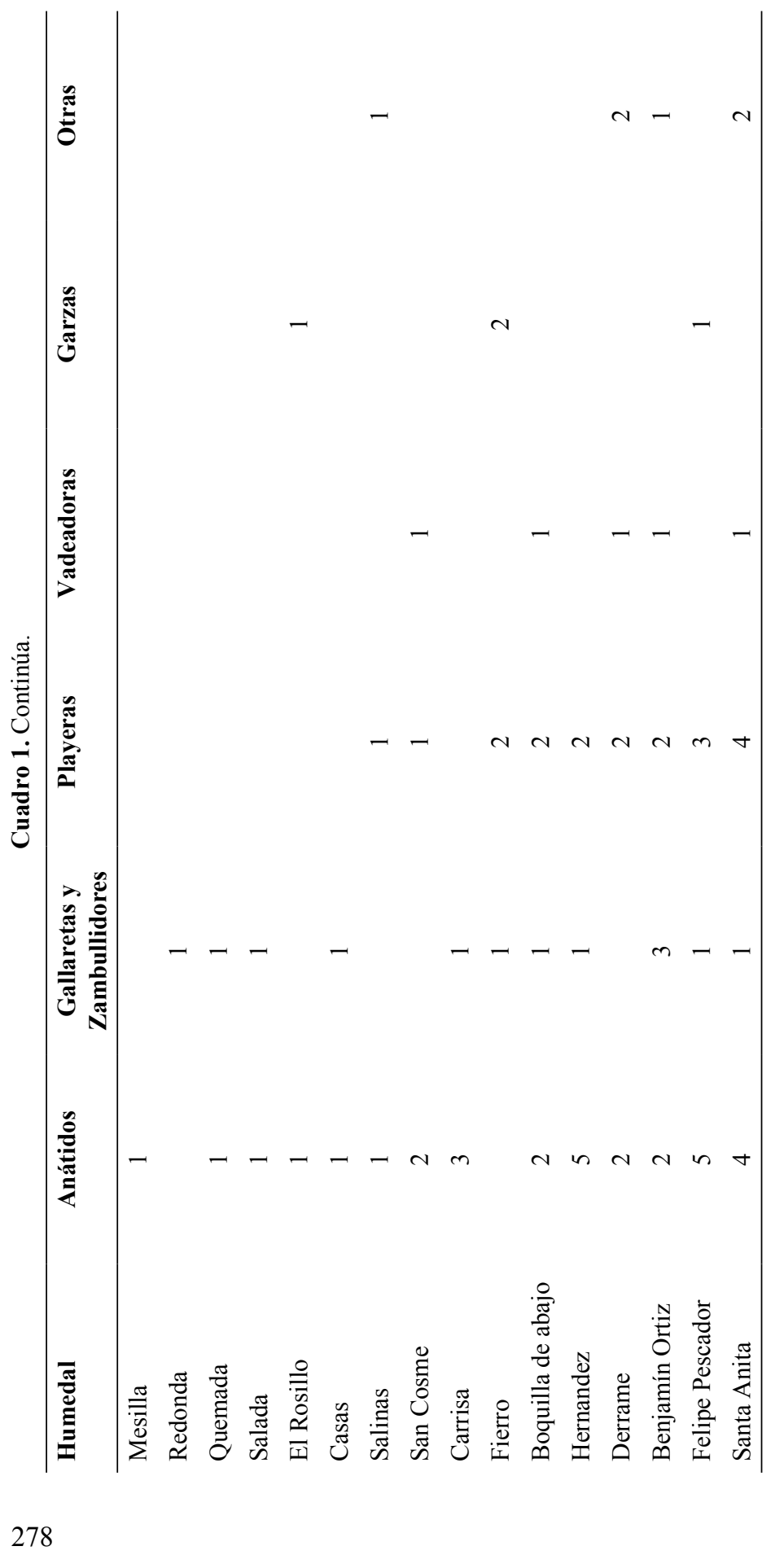


Cuadro 2. Correlación de presencia de grullas y riqueza de especies.

No se encontraron correlaciones significativas $p<0.05$.

\begin{tabular}{lcccc}
\hline Variable & Media & Desv. Std. & Presencia & Riqueza \\
\hline Presencia & 0.51 & 0.50 & 1.00 & 0.16 \\
Riqueza & 4.97 & 3.32 & 0.16 & 1.00 \\
\hline
\end{tabular}

Cuadro 3. Correlación de Abundancia de grullas con riqueza de especies. No se encontraron correlaciones significativas $p<0.05$

\begin{tabular}{lrccc}
\hline Variable & Media & Desv. Std. & Abundancia & Riqueza \\
\hline Abundancia & 338.46 & 807.23 & 1.00 & 0.03 \\
Riqueza & 4.97 & 3.33 & 0.03 & 1.00 \\
\hline
\end{tabular}

Cuadro 4. Correlación de Abundancia por categoría y riqueza de especies. No se encontraron correlaciones significativas $p<0.05$

\begin{tabular}{lcccc}
\hline Variable & Media & Desv. Std. & $\begin{array}{c}\text { Abundancia } \\
\text { Categoría }\end{array}$ & Riqueza \\
\hline $\begin{array}{l}\text { Abundancia } \\
\text { Categoría }\end{array}$ & 1.97 & 3.32 & 1.00 & 0.14 \\
Riqueza & 4.98 & 3.33 & 0.14 & 1.00 \\
\hline
\end{tabular}

Cuadro 5. Correlación de presencia de grullas y riqueza de patos y zambullidores, vadeadoras y otras. No se encontraron correlaciones significativas $p<0.05$

\begin{tabular}{lcccccc}
\hline Variable & Media & Desv. Std. & Presencia & $\begin{array}{c}\text { Patos y } \\
\text { Zambullidores }\end{array}$ & Vadeadoras & Otras \\
\hline Presencia & 0.51 & 0.50 & 1.00 & 0.28 & 0.05 & -0.02 \\
Patos y & 2.46 & 1.57 & 0.29 & 1.00 & & \\
Zambullidores & & & & & 1.00 & \\
Vadeadoras & 1.70 & 1.76 & 0.05 & & & 1.00 \\
Otras & 0.81 & 0.81 & -0.02 & & & \\
\hline
\end{tabular}

Se observó una asociación en la riqueza de especies entre los humedales con presencia de grulla, ya que algunas especies sólo están presentes cuando están las grullas. Lo anterior puede deberse a una regla de asociación (ansembly rules) si consideramos cada humedal como una isla en la que los recursos son limitados, lo que excluye ciertas especies que compiten por los mismos recursos, mientras que para otras se pueden asociar debido a que hay menor presión entre las mismas, aunque la forma de toma de datos no permiten asegurarlo (Connor \& Simberloff 1979, Gotteli \& McCabe 2002). 
López-Saut et al.: La grulla como indicador de aves acuáticas

Cuadro 6. Humedales con una similitud mayor a 0.60 . Con * los humedales en los que se registraron grullas.

\begin{tabular}{lc}
\hline Humedales similares & Índice Whittaker \\
\hline Carriza - Tordillo* & 0.61 \\
Carriza - Santiaguillo* & 0.66 \\
Carriza - Quemada & 0.66 \\
Redonda - Quemada & 0.66 \\
Redonda - Casas & 0.66 \\
Fierro - Bordo Rosillo & 0.66 \\
Fierro - Salada* & 0.66 \\
Fierro - San Bartolo* & 0.66 \\
Casas- Carriza & 0.66 \\
Salitral* - San Bartolo & 0.66 \\
Salitral* - Salada* & 0.66 \\
Salitral* - Bordo Rosillo & 0.66 \\
Bordo Rosillo - Virgen* & 0.66 \\
Nuestra Señora* - San Bartolo* & 0.66 \\
La Esperanza* - Villa Hidalgo* & 0.72 \\
Bustillos* - Casas & 0.66 \\
Seca-Santiaguillo* - San Juan de Ahorcado* & 0.68 \\
Saniaguillo* - Virgen* & 0.66 \\
Nariz* - Ojo Federico* & 0.66 \\
\hline
\end{tabular}

Algunas de estas especies son Anas platyrhynchos y A. p. diazi y Aythya americana en el caso de los anátidos y Calidris mauri en los playeros. Aunque la dieta y hábitos de estas especies son distintos, los requerimientos del tipo de humedal pueden ser similares a los de la grulla gris. También se reportó Phalacrocorax brasilianus en humedales con grullas. Estas asociaciones pueden deberse a la estructura del humedal requerida que pueden ser áreas pocos profundas para las grullas, y pueden tener a la vez áreas más profundas para especies como los cormoranes. No obstante, se puede ver que las asociaciones no son con gremios de especies, sino con especies particulares. Estas relaciones pueden no estar relacionadas a un recurso en común, pero sí puede deberse a la estructura de los humedales lo que podría asociarse a la calidad del hábitat.

Las grullas están presentes tanto en los humedales permanentes como en los temporales, estos últimos sirve a la llegada de las aves migratorias por tener aún agua y producción de alimento. Los humedales permanentes son más fáciles de identificar y 


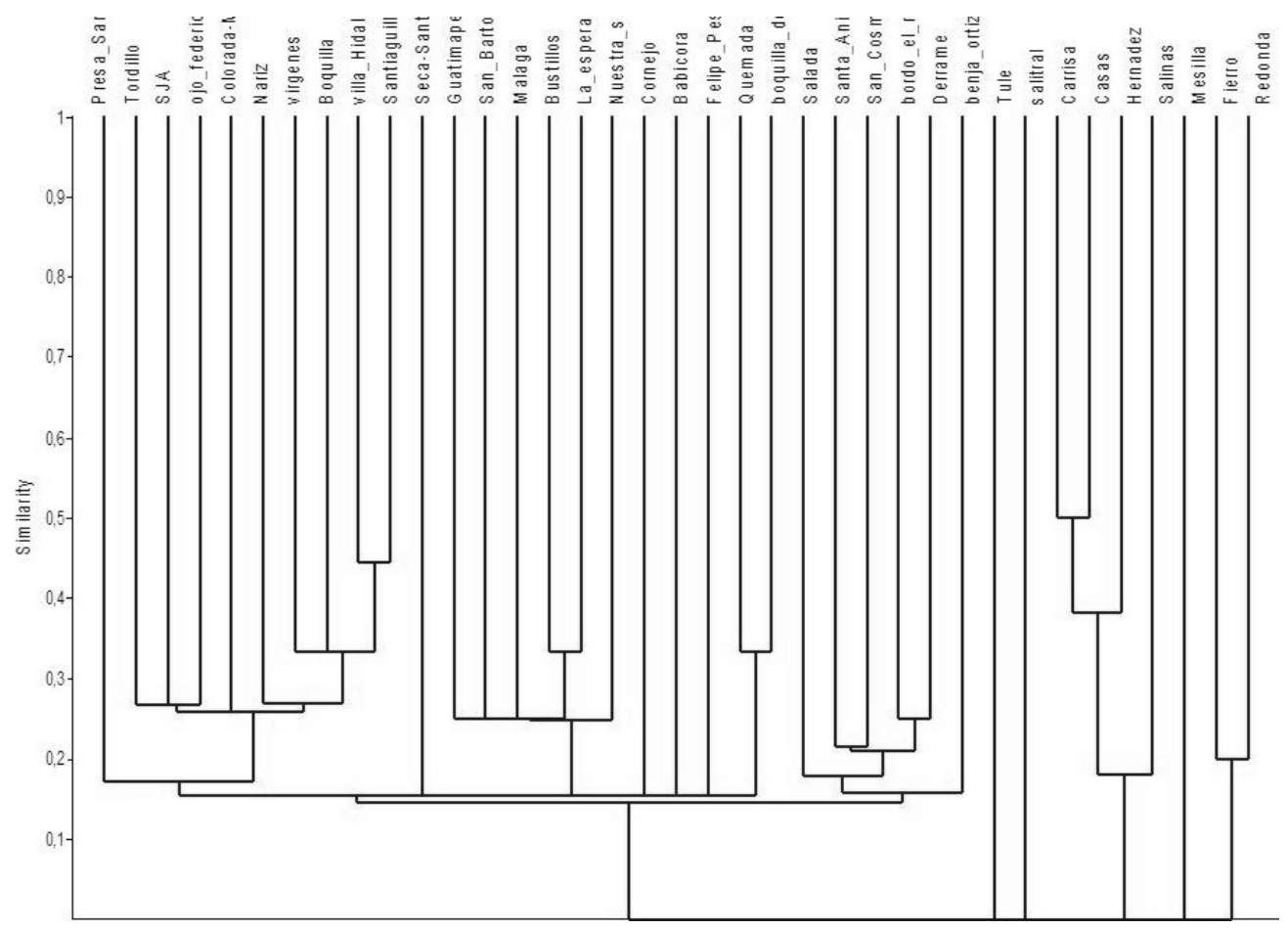

Figura 3. Índice de similitud de Jaccard. Se analizaron 38 humedales con este índice y no presentaron similitud alta entre sí en cuanto a la composición de especies.

clasificar, por lo que es más simple evaluar su estado para la conservación de servicios ambientales y ecológicos. En cambio los humedales temporales de las zonas desérticas presentan el problema de identificación y clasificación (Cowardin et al. 1979). En el Desierto Chihuahuense los humedales temporales son zonas con baja pendiente y superficie variable, se caracterizan por tener una llanura de inundación que se satura a la llegada de las lluvias de finales de verano y que ve modificada dependiendo de la cantidad de precipitaciones (Evenari et al. 1985, Macmahom \& Wagner 1985, Grünberger 2004). Dependiendo de la intensidad y los ciclos de lluvia los humedales pueden no formarse o bien permanecer largas temporadas, hasta mediados de invierno e inclusive en ocasiones hasta finales de invierno. Cuando se forman estos humedales presentan una gran cantidad de invertebrados, debido a la ausencia de depredadores residentes. Se convierten en las presas de especies que aprovechan ese nuevo recurso, entre las que se encuentran las aves migratorias (NRCS 2007). Para ellas los humedales tienen un papel importante como zonas de descanso (stopovers) durante la migración y para pasar el invierno. Los humedales temporales son hábitats 
López-Saut et al.: La grulla como indicador de aves acuáticas

Cuadro 7. Matriz biológica propuesta por González et al. (2003). Los valores fueron tomados a partir del conocimiento de la grulla gris de Tacha et al. (1994), Archibald \& Maine (1996), Iverson et al.

(1992), Lopez-Saut (2011).

\begin{tabular}{cccccc}
\hline $\begin{array}{c}\text { Estado de } \\
\text { Conservación }\end{array}$ & Endemismo & Distribución & $\begin{array}{c}\text { Historia } \\
\text { Natural }\end{array}$ & $\begin{array}{c}\text { Especialización } \\
\text { a un hábitat }\end{array}$ & Total \\
\hline 1 & 0.5 & 4 & 4 & 4 & $\mathbf{1 3 . 5}$ \\
\hline
\end{tabular}

Cuadro 8. Matriz Manejo propuesta por González et al. (2003). Para el calculo de los valores se utilizaron los datos de Drewien et al. 1996, Kinzel et al. 2006, Melvin \& Temple 1983, Nesbitt \&

Williams 1990, Krapu et al. 2011.

\begin{tabular}{cccc}
\hline $\begin{array}{c}\text { Posibilidad de } \\
\text { muestreo }\end{array}$ & $\begin{array}{c}\text { Posibilidad de } \\
\text { manipulación }\end{array}$ & $\begin{array}{c}\text { Posibilidad de } \\
\text { determinación }\end{array}$ & Total \\
\hline 4 & 4 & 4 & $\mathbf{1 2}$ \\
\hline
\end{tabular}

importantes e inclusive cruciales, si están disponibles, hasta para un $80 \%$ de las aves acuáticas (Caziani et al 2001). Cuando estos humedales desaparecen o son alterados se ha reportado un declive de las poblaciones de patos de entre el 20 al 60\% (NRCS 2007). Por su misma temporalidad son sensibles a la perturbación humana pudiendo inclusive desaparecer por un mal manejo (NRCS 2007). Por ejemplo, según expertos en el Desierto Chihuahuense, en particular en Zacatecas, se ha reportado que la perforación de nuevos pozos para extracción de agua para las ciudades ha afectado la temporalidad de los humedales y disminuido el nivel de agua de los permanentes (Miguel Angel Díaz Castorena, com. pers.). Por lo anterior, el que las grullas estén relacionadas con la riqueza de aves, principalmente migratorias que usan estos sitios, hace que puedan ser bioindicadores importantes de humedales temporales que requieran un mejor manejo para su conservación.

El número de especies puede reflejar el estado del humedal en cuanto a la disponibilidad de alimento y agua, así como su estructura si encontramos varios gremios dentro del mismo. Aunque no medimos en este estudio estos parámetros del humedal, las especies que encontramos pueden ser un reflejo de esta condición (Bayard \& Elphik 2010). Guadagnin et al. (2005) ha reportado que los humedales aislados y fragmentados tienen una correlación negativa con la riqueza de especies y su abundancia. La variación en el número de especies entre los sitios con grullas y sin grullas puede estar relacionada con los patrones migratorios y las temporadas cuando se realizan los muestreos que hace que fluctué el número de especies registradas en el humedal. Pero no debemos descartar que las aves sirvan para evaluar el estado de salud de los ecosistemas basados en aspectos sociales (interacción intraespecíficas) y en la presencia de otras especies o grupos de aves (interacciones interespecíficas) (Reed \& Dobson 1993, Danchin \& Warner 1997, Bayard \& Elphik 2010). Guadagnin et al. (2005) 
encontraron que en sitios fragmentados las especies ausentes son aquellas con mayor masa corporal, principalmente los anátidos y especies raras como las garzas que en este estudio no se encontraban en los humedales. Aunque no estudiamos el efecto de la fragmentación y aislamiento de los humedales, se encontró que en aquellos humedales donde estuvieron presentes las grullas también mostraron una mayor presencia de anátidos y garzas, lo que puede indicar que los humedales donde se presentan las grullas pueden tener un mejor estado de conservación o de salud, lo cual puede ser evaluado usando el número de individuos y las especies a través de la presencia y abundancia de otras especies de aves acuáticas. Aunque en este estudio no se analizaron estas posibilidades y se encontró poca correlación entre las mismas.

En el caso de los playeros (Charadriiformes) se ha reportado que disminuyen su diversidad en los fragmentos más alejados (Guadagnin et al. 2005). Disminuyó el número de especies en los lugares donde se registraron las grullas, lo que siguiendo a Guadagnin et al. (2005) podría indicar que el hábitat está fragmentado. No obstante, si consideramos que las especies de playeros que se presentaron en los humedales son especies residentes en México, es posible que no se encuentre una asociación en los sitios que prefieren las aves migratorias, como son las grullas. Por otro lado, se observa un mayor número de especies vadeadoras en donde están presentes las grullas, y estas son especies migratorias y con una mayor talla corporal que las playeras, las que pueden servir como vigías para avisar de peligros en los alrededores. Se ha encontrado que algunas especies de zambullidores y las gallaretas son aves que en áreas urbanas se adaptan muy bien a humedales con baja calidad y con disturbios (Traut \& Hostetler 2003), lo que podría explicar su presencia en lugares donde no se encuentran las grullas y su ausencia en los humedales donde están presentes.

Basados en lo propuesto por González et al. (2003) la grulla gris cumple con siete de los ocho criterios que debe tener un ave bioindicadora ya que es una especie bajo protección especial en México (SEMARNAT 2010) de distribución amplia en el desierto Chihuahuense (López-Saut et al.2011), se conoce su historia natural ya que está bien estudiada y se conocen sus patrones migratorios, reproductivos, dieta e inclusive requerimientos nutricionales (Walkinshaw 1973, Reinecke y Krapu 1986, Tacha et al. 1992, Ballard \& Thompson 2000). En los Estados Unidos de Norteamérica se han realizado diversos estudios sobre el hábitat invernal (Iverson et al. 1985), alimentación (Ballard \& Thompson 2000) y conducta (Harvey et al 1968, Tacha et al. 1988) que nos permiten interpretar las observaciones que se hagan en México para un manejo de las grullas en México. Por lo anterior las grullas son buenos bioindicadores, además de poder ser usada como especie carismática, bandera y sombrilla para los humedales del desierto, como han servido en otras áreas del mundo (von Treuenfels 2006).

Aunque existen pocos trabajos sobre las grullas en México principalmente en Chihuahua (A. Lafón-Terraza, com. pers.) se tiene la información suficiente para iniciar 
un monitoreo de las grullas en el Desierto Chihuahuense y poder interpretar la información para la conservación de los humedales. Un ejemplo de lo anterior es el presente estudio, que forma parte de otro proyecto donde se está generando información sobre el hábitat de las grullas en México para su conservación.

En términos de detección, las grullas pueden ser observadas desde transectos aéreos (Drewien et al. 1996, Kinzel et al. 2006), lo que permitiría evaluar una gran área de los humedales en el Desierto Chihuahuense. Una vez identificados los humedales, se puede realizar una evaluación por tierra. Su conducta gregaria durante el invierno las hace muy fáciles de observar e identificar, asimismo, las grullas dejan rastros muy claros de su presencia en los humedales, como son las huellas y plumas (ChávezRamírez 2003, López-Saut et al. 2011). Las grullas han sido anilladas y colocado transmisores convencionales y satelitales para determinar sus movimientos migratorios, uso del hábitat y distribución con buenos resultados (Melvin \& Temple 1983, Nesbitt \& Williams 1990, Krapu et al. 2011), además se le pueden realizar estudios parasitológicos y de condición para seguir a las poblaciones presentes en los humedales (Olsen et al. 1996).

En conclusión las grullas pueden ser usadas como indicadores de la composición de especies (a nivel de grupos) y la riqueza de aves acuáticas, con lo cual nos puede ser servir como indicadores ambientales del estado de los humedales, aunque es necesario realizar un estudio más profundo. Asimismo, su relación con humedales temporales puede ayudar su correcto manejo, ya que sin importar su temporalidad, si encontramos indicios de ser usados por las grullas, serían sitios importantes para las aves migratorias acuáticas, por lo que deberían ser considerados para un manejo adecuado y conservación. Si se implementa esta estrategia permitiría evitar cambio de usos del suelo, aun cuando no estén presentes los humedales por los periodos de lluvia y sequía. Proponemos que mediante un programa de monitoreo se use la presencia o indicios de presencia de las grullas como un indicador de la calidad, temporalidad y grado de conservación de los humedales del desierto Chihuahuense, lo que permitirá hacer mejores programas de manejo sobre todo de los humedales temporales.

AGRADECIMIENTOS. Agradecemos a Profauna-Chihuahua y SEMARNAT-Zacatecas, especialmente a J. Macias-Patiño y M. A. Castorena. Al soporte financiero proporcionado por Platte River Whooping Crane Maintenance Trust y al proyecto SEP-CONACYT 155956, a CONACYT por la beca de doctorado número 162952 a EGLS, al Centro de Investigaciones Biológicas el Noroeste.

\section{LITERATURA CITADA}

Aguilar, V. 2003. Aguas continentales y diversidad biológica de México: un recuento anual. Biodiversitas, 48: 1-15.

Arizmendi M. C. \& Márquez, L. 2000. Áreas de importancia para la conservación de las aves en México. CONABIO. México. 
Ballard B. M. \& Thompson, J. E. 2000. Winter diets of Sandhill cranes from central and coastal Texas. The Willson Bulletin, 112: 263-268.

Barceló, I., Guzmán-Aranda, J. C., Chávez-Ramírez, F. \& Powell, L. K. 2012. Rural Inhabitant Perceptions of Sandhill Cranes in Wintering Areas of Northern Mexico. Human Dimension of Wildlife, 17: 301-307.

Bayard T. S. \& Elphik, C. S. 2010. Using spatial point-pattern assessment to understand the social and environmental mechanisms that drive avian habitat selection. The Auk, 127: 485-494.

Canadian Wildlife Service and U.S. Fish and Wildlife Service. 2005. Draft International recovery plan for the whooping crane. Ottawa: Recovery of Nationally Endangered Wildlife (RENEW), and U.S. Fish and Wildlife Service, Albuquerque, New Mexico.

Caziani, S. M., Derlindati, E. J., Talamo, A., Sureda, A. L., Trucco, C. E. \& Nicolossi, G. 2001. Waterbird richness in altiplano wetlands of northwestern Argentina. Waterbird, 24: 103-117.

Chavez-Ramirez, F. 2003. New locations and range extension of wintering Sandhill cranes in central northern Mexico. Proceedings North American Crane Workshop, 9: 164-174.

CONAGUA. 2007. Estadísticas del agua en México. CNA. México.

CONAGUA. 2011. Estadísticas del agua en México. CNA. México.

CONANP 2010. Humedales de México. Sitios RAMSAR. En línea: http://ramsar.conanp.gob.mx/sitios. php Visitado: 10 de abril 2002.

Connor, E. F. \& Simberloff, D. 1979. The assembly of species communities: Chance or competition? Ecology, 60: 1132-1140.

Contreras, A. J., García, J. A., Guzmán, A. \& González Rojas, J. I. 2001. Aprovechamiento de las aves cinegéticas, de ornato y canoras de Nuevo León México. Ciencia UANL, 4: 462-469.

Cooper, A., Shinea, T., McCanna, T. \& Tidane, D. A. 2006. An ecological basis for sustainable land use of Eastern Mauritanian wetlands. Journal of Arid Environments, 67:116-141.

Cowardin, L. M., Carpenter, V., Golet, F. C. \& LaRose, E. T. 1979. Classification of wetlands and deepwater habitat of United States. U. S. Departament of the interior, Fish and Wildlife Service, Washington, D. C. Jamestown, ND: Northern Prairie Wildlife Research Center Home Page. http:// www.npwrc.usgs.gov/resource/wetlands/classwet/ (Version 04DEC98) 23 Febrero 2014.

Danchin, E. \& Warner, R. H. 1997. The evolution of coloniality: the emergence of new perspectives. Trends in Ecology and Evolution, 12: 342-347.

Drever, M. C., Aitken, K. E. H., Norris, A. R. \& Martin, K. 2008. Woodpeckers as reliable indicators of bird richness, forest health and harvest. Biological Conservation, 141: 624-634.

Drewien, R. C., Brown, W. B. \& Benning, D. S. 1996. Distribution and abundance of Sandhill cranes in Mexico. The Journal of Wildlife Management, 60: 270-285.

Evenari, M., Noy-Meir, I., \& Goodall, D. W. (editores) 1985. Hot Deserts and Arid Shrublands, A. (Ecosystems of the World 12A). Elsevier, Amsterdam.

Fleishman, E., Thomson, J. R., MacNally, R., Murphy, D. D. \& Fay, J. P. 2005. Using indicator species to predict species richness of multiple taxonomic groups. Conservation Biology, 19: 11251137.

Frazier, S. 1999. Ramsar sites overview. A synopsis of the world's wetlands of international importance. Wetlands International. The Works, Newbury, Berkshire, UK

González, M. A., Guzmán, J., Martin, M. F. \& Domínguez, L. E. 2003. Un método para la selección de aves bioindicadoras con base en sus posibilidades de monitoreo. Huitzil, 4: 10-16.

Gotteli, N. J. \& McCabe, D. J. 2002. Species co-occurrence: A meta-anlysis of J. M. Diamond's assembly rules mode. Ecology, 83: 2091-2096.

Grünberger, O. 2004. El ecosistema playa, pp. 32-40. In: O. Grünberger, V. M. Reyes-Gómez, J.L. Janeau. Las playas del desierto Chihuahuense (parte mexicana). Influencia de las sales en ambientes 
López-Saut et al.: La grulla como indicador de aves acuáticas

áridos y semiáridos. Coedición L'Institut de Recherche pour le Développement, (IRD) y el Instituto de Ecología, A.C. (INECOL).

Guadagnin, D. L., Peter, A. S., Carvalho, L. F. \& Maltchik, L. 2005. Spatial and temporal patterns of waterbird assemblages in fragmented wetlands of southern Brazil. Waterbird, 28: 261-272.

Harvey, J. M., Lieff, B. C., MacInnes, C. D. \& Prevett, J. P. 1968. Observation of behavior of Sandhill cranes. The Wilson Bulletin, 80: 421-425.

Hinojosa-Huerta, O. DeStefano, S., Carrillo-Guerrero, Y. \& Valdés, S. W. 2004 Waterbird communities and associated wetlands of the Colorado River delta, Mexico. Studies in Avian Biology, 27: $52-60$.

Howell S., N. G. \& Webb, S. 1995. A guide to the birds of Mexico and Northern Central America. Oxford University Press, USA.

Iverson, G. C., Vohs, P. A. \& Tacha, T. C. 1985. Habitat use by Sandhill cranes wintering in Texas. The Journal of Wildlife Management, 49: 1074-1082.

Karr, J. R. 1991. Biological integrity: a long-neglected aspect of water resource management. Ecological applications, 1: 66-84.

Kinzel, P. J., Nelson, J. M., Parker, R. S. \& Davis, L. R. 2006. Spring census of mid-continent Sandhill Cranes using aerial infrared videography. Journal of Wildlife Management, 70: 70-77.

Krapu, G. L., Brandt, D. A., Jones, K. L. \& Johnson, D. H. 2011. Geographic distribution of midcontinent population of Sandhill cranes and related management applications. Wildlife Monographs, 175: 1-38.

León-Portilla, G. 1999. La visión de los vencidos. UNAM México.

López-Saut, E. G., Chávez-Ramírez, F. \& Rodríguez-Estrella, R. 2011. New Records of Wintering Grounds for Sandhill Cranes in Mexico. Waterbirds, 34: 239-246.

Macmahon, J. A. \& Wagner, F. H. 1985. The Mojave, Sonoran and Chihuahuan desert of North America, pp. 105-202. In: Evenari, M., Noy-Meir, I., y Goodall, D.W. (Eds.). Hot Deserts and Arid Shrublands, A. (Ecosystems of the World 12A). Elsevier, Amsterdam.

Meine, C. D. \& Archibald, G. W. (Comps.) (1996). The cranes: status survey and conservation action plan. Gland, Switzerland y Cambridge, UK: IUCN SSC Crane Specialist Group. Gland and Cambridge, UK.

Melvin, S. M. \& Temple, S. A. 1983. Fall migration and mortality of interlake, Manitoba Sandhill cranes in North Dakota. Journal of Wildlife Management, 47: 805-817.

Mikusiński, G., Gromadzki, M. \& Chylarecki, P. 2001. Woodpeckers as indicators of forest bird diversity. Conservation Biology, 15: 208 $\square 217$.

Morgan, N. C. \& Boy, V. 1982. An ecological survey of standing waters in North West Africa: 1. Rapid survey and classification. Biological Conservation, 24: 5-44.

Nesbitt, S. A. \& Williams, K. S. 1990. Home range and habitat use of Florida Sandhill cranes. Journal of Wildlife Management, 54: 92-96.

NRCS. 2007. Temporarily flooded wetlands. Fish and Wildlife Management, Leaflet 47: 1-10.

Okes N. C., Hockey, P. A. R. \& Cumming, G. S. 2008. Habitat use and life history as predictor of bird response to habitat change. Conservation Biology, 22: 151-162.

Olsen, G. H., Langenberg, J. A. \& Carpenter, J. W. 1996. Medicine and surgery, pp. 137-174. In: D. H. Ellis, G. F. Gee y C. M. Mirande (Eds.). Cranes: Their biology, husbandry, and conservation. National Biological Service/International Crane Foundation Limited Edition. USA.

Rappole J. H., King, D. I. \& Diez, J. 2003.Winter- vs breeding-habitat limitation for an endangered avian migrant. Ecological Application, 13: 735-742.

Reed, J.M. \& Dobson, A.P. 1993. Behavioural constraints and conservation biology: Conspecific attraction and recruitment. Trends in Ecology and Evolution, 8: 253-256. 
Reinecke, K. J. \& Krapu, G. L. 1986. Feeding ecology of Sandhill cranes during spring migration in Nebraska. Journal of Wildlife Management, 50: 71-79.

Samraoui, B. \& Samraoui, F. 2008. An ornithological survey of Algerian wetlands: Important Bird Areas, Ramsar sites and threatened species. Wildfowl, 58: 71-96.

Sánchez, O., Herzig, M., Peters, E., Márquez, R. \& Zambrano, L. (Ed). 2007. Perspectivas sobre conservación de ecosistemas acuáticos en México. Secretaria del Medio Ambiente y Recursos Naturales-Instituto Nacional de Ecología. México.

SECTUR. Estudio Estrátegico de viabilidad del segmento de turismo cinegético en México. Sistesis Ejecutiva. En línea:

http://cestur.sectur.gob.mx/pdf/estudioseinvestigacion/segmentosyproductos/productos/Turismo_Cinegetico.pdf Visitado (02 marzo 2014)

SEMARNAP. 1999. Acuerdo por el que se establece el calendario de aprovechamiento cinegético. En línea: http://www.iaconsma.com/iaconsma_sys/normatividad/Acuerdo16.pdf. Visitado (02 marzo 2014)

SEMARNAT. 2009. Informe de la situación del medio ambiente en México. Edición 2008. Compendio de Estadísticas Ambientales. México.

SEMARNAT. 2010. Norma Oficial Mexicana NOM-059-ECOL-2010, protección ambiental- Especies nativas de México de flora y fauna silvestre -Categorías de riesgo y especificaciones para su inclusión, exclusión o cambio. Lista de especies de riesgo. México.30 de Marzo 2010.

Sharp, D. E., Kruse, K. L. \& Dubovsky, J. A. 2006. Status and harvests of sandhill cranes: Mid-Continent and Rocky Mountain Populations. Unnumbered. Administrative Report, U.S. Fish and Wildlife Service, Denver, Colorado.

Sokal R. R. \& Rohlf, F. J. 1994. Biometry: The principle and practices of statistic in biological research. 3th edition. W.H Freeman, New York.

Springate-Baginski O., Allen, D. \& Darwall, W. R. T. (Eds.). 2009. A integrate wetland assessment toolkit: A guide to good practice. Gland Suecia: IUCN and Cambridge, UK: IUCN Species Programme.

Tacha, T. C., Nesbitt, S. A. \& Vohs, P. A. 1992. Sandhill crane, pp. 24. In: Poole A., P Stettenheim \& F. Gill (Eds.). The birds of north America. Washington, D. C. Philadelphia. The Academy of Natural Sciences.

Tankersley Jr., R. D. 2004. Migration of birds as an indicator of broad-scale environmental condition. Environmental Monitoring and Assessment, 94: 55-67.

Toledo, A. 2006. Agua, hombre y paisaje. Secretaría de Medio Ambiente y Recursos Naturales-Instituto Nacional de Ecología. México.

Traut, A. H. \& Hostetler, M. E. 2003. Urban lakes and waterbirds: Effect of development on avian behavior. Waterbirds, 26: 290-302.

Verbist, K., Santibañez, F., Gabriels, D. \& Soto, G. 2010. Atlas de Zonas Áridas de América Latina y El Caribe. CAZALAC. Documentos Técnicos del PHI-LAC. http://www.atl.org.mx/seminario/images/docs/ATLAS_de_Zonas_Aridas_de_ALC_final_light.pdf (23 febrero 2014)

von Treuenfels C.A. 2006. The magic of cranes. Harry N. Abrams. New York.

Walkinshawn, L. 1973. Cranes of the world. Winchester Press, New York.

Whittaker, R. H. 1975. Communities and ecosystems. MacMillan Publishing Co., Inc., New York. 